\title{
STATUS OF THE LASER MASTER OSCILLATOR SYSTEM AT FLASH
}

\author{
A. Winter, P. Schmüser, Universität Hamburg, Hamburg, Germany, \\ F. Loehl, H. Schlarb, DESY Hamburg, Germany, \\ W. Jalmuzna, Warzaw University
}

\begin{abstract}
An optical synchronization system based on the timingstabilized distribution of ultra-short optical pulses has been proposed for next generation light sources, e.g. the European XFEL. The concept will be implemented and tested at FLASH at DESY. This paper describes the status of the optical master oscillator, which consists of a two mode-locked Erbium-doped fiber lasers running in parallel.
\end{abstract}

\section{INTRODUCTION}

One of the key challenges for X-ray free electron lasers is to implement an RF timing and synchronization system with an accuracy in the order of 10 femtoseconds that allows to fully exploit the narrow width of the X-ray pulses for time-resolved experiments.

These demanding requirements cannot be met by conventional RF distribution systems based on microwave oscillators and semi-rigid coaxial cables since the distances involved are on the order of kilometers. A promising alternative is an optical system [1]. A periodic train of subpicosecond light pulses is generated in a mode-locked fiber laser and distributed along the machine through fibers with optical length stabilization. The synchronization information is contained in the precise repetition frequency of the pulse train.

A mode-locked laser serves as an ultra-stable laser master oscillator (LMO) for the proposed optical synchronization system for the European XFEL, which will be tested at the FEL facility FLASH [2]. Fiber lasers are well suited to realize such an optical master oscillator, because of the ease of coupling to the fiber distribution system, their excellent long-term stability, and the well-developed and mature components that are available at the optical communications wavelength of $1550 \mathrm{~nm}$. A detailed description of the Erbium-doped fiber laser can be found elsewhere [3].

\section{CHOICE OF REPETITION RATE OF THE LMO SYSTEM}

There are two principal choices for the repetition rate of the LMO system. Stretched-pulse EDFLs can operate at repetition rates between $20 \mathrm{MHz}$ and $100 \mathrm{MHz}$, due to dispersion constraints in the optical fiber. A possibility to increase the repetition rate is to construct a soliton laser [4]. This type of laser, looking similar in component design to the stretched-pulse version, has no dispersion constraints limiting tolerable fiber length, only practical considerations 07 Accelerator Technology Main Systems limit the repetition rate to $<250 \mathrm{MHz}$. For FLASH, the repetition rate needs to be a sub-multiple of the accelerator $\mathrm{RF}$ of $1300 \mathrm{MHz}$. This makes values of $54 \mathrm{MHz}, 108 \mathrm{MHz}$ or $216 \mathrm{MHz}$ feasible choices.

There are various merits and drawbacks to either choice. A definite advantage of the higher repetition rate is the ease of alignment of stabilized fiber links based on optical crosscorrelation [5]. For these fiber links, it is imperative to balance the dispersion in each link by using suitable combinations of fibers with positive and negative dispersion and to assure that pulses returning through the link can be overlapped in the cross correlator with pulses directly from the laser. This can be achieved by delaying one arm with respect to the other. For a repetition rate of $54 \mathrm{MHz}$ this requires a maximum delay of $9.25 \mathrm{~ns}$ or almost 3 meters in air, making the mechanical construction of the cross correlator rather cumbersome. At a repetition rate of $216 \mathrm{MHz}$, a delay of only $77 \mathrm{~cm}$ is needed. A further benefit of the high repetition rate is that for applications where an overlap with the electron bunch is required, the residual delay that needs to be introduced at the end of a fiber link is smaller. The drawback of a high repetition rate lies in the amplitude detection of pulses in for instance electron bunch arrival time measurements [6]. ADC sampling rates in excess of $400 \mathrm{MHz}$ are required. This is possible, but at a reduced dynamic range. Furthermore, optical gating is required to generate a lower repetition rate for applications where high optical pulse energy is required, for instance for the locking of other lasers around the facility.

One of the most important features of the LMOs is their low phase noise at high offset frequencies. Erbium-doped fiber lasers exhibit an extremely low intrinsic phase noise making these lasers competitive with the best low-noise microwave oscillators. However, environmental effects like microphonics and vibrations cause excessive lowfrequency phase noise, which can be significantly reduced by phase-locking the fiber laser to a ultra-low noise microwave oscillator. This is done by comparing the phase of the reference oscillator to the phase of a suitable harmonic of the LMO repetition rate, which is selected by a bandpass filter after photo detection of the optical pulse train. This error signal is amplified by a PI controller and fed to a piezo-based fiber stretcher, onto which a substantial part of the optical fiber making up the laser cavity is wound, or alternatively to a piezo-mirror within the laser cavity, adjusting the repetition rate of the LMO.

A phase noise measurement for both laser types (stretchedpulse and soliton) is shown in 1. Both exhibit excellent performance, with negligible performance differences in the 
regime above $1 \mathrm{kHz}$. The noise floor of the soliton laser is lower, as the measurements are limited by the electrical power level in the filtered harmonic of the laser repetition rate after photodetection. Since the total output power of the photodiode is constant, there is more power in each spectral line of the higher repetition rate laser. So from the high-frequency noise point of view, both laser systems are potential candidates for the LMO system.

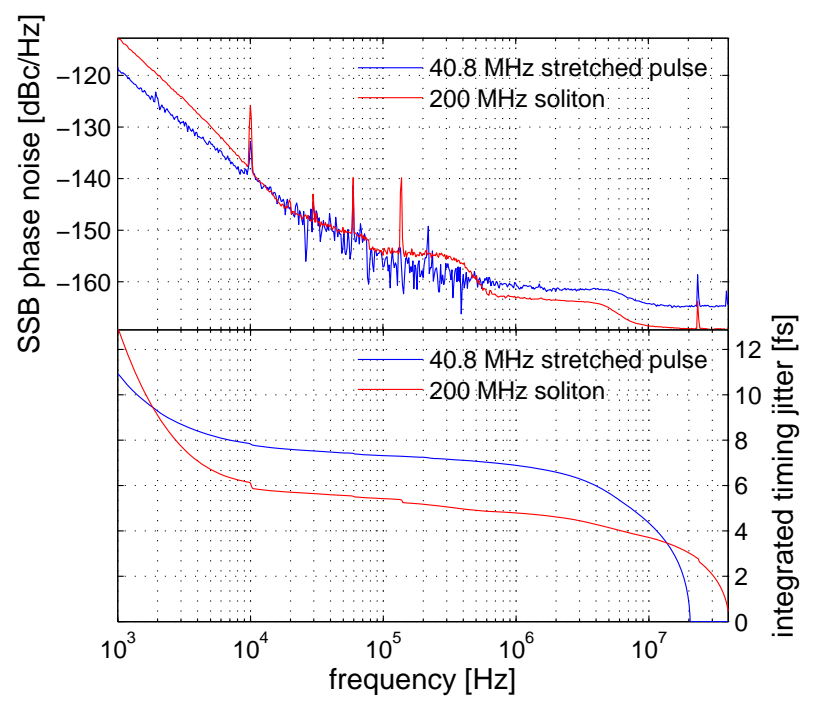

Figure 1: Phase noise comparison between a $40.8 \mathrm{MHz}$ stretched-pulse laser and a $200 \mathrm{MHz}$ soliton laser. Top: single-sideband phase noise measured at the harmonics at $980 \mathrm{MHz}$ (stretched-pulse) and $1.2 \mathrm{GHz}$ (soliton), respectively. Bottom: integrated timing jitter starting integration at $20.4 \mathrm{MHz}$ (stretched-pulse) and $40 \mathrm{MHz}$ (soliton).

\section{LAYOUT OF THE LMO SYSTEM}

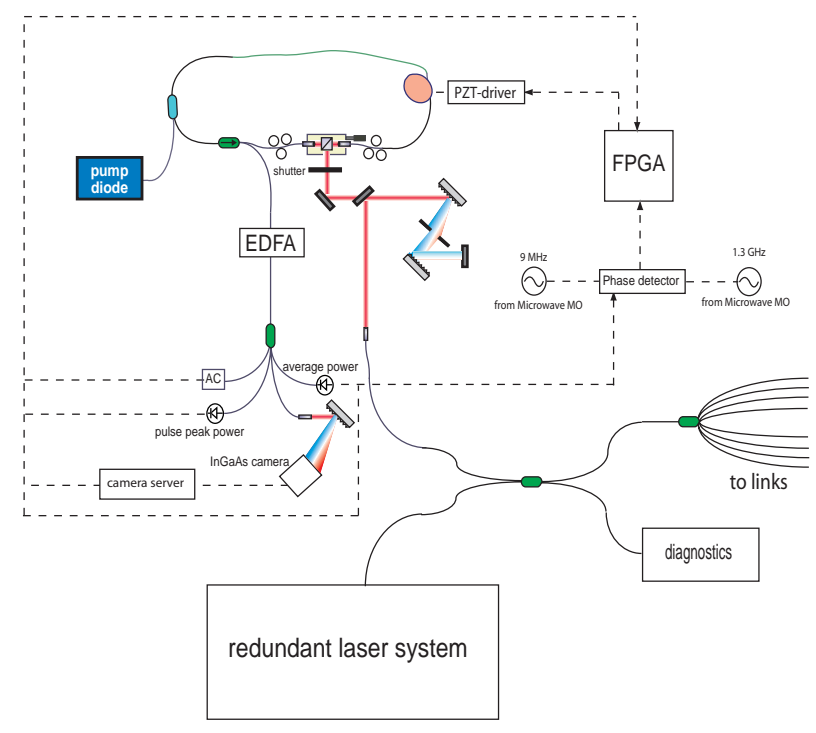

Figure 2: Schematic of the proposed FLASH LMO system
A schematic layout of the LMO system for FLASH is shown in Figure 2. To achieve the required uptime of the LMO system, it is built redundant. Two lasers of repetition rate of $54 \mathrm{MHz}$ or $216 \mathrm{MHz}$ run continuously, and should one of the lasers fail, the backup unit will take over. There is substantial diagnostics involved to ensure proper operation of the fiber lasers, which will be described in the next section. The stretched-pulse fiber lasers require some spectral narrowing as the nominal spectral width is larger than desired. This is done by an iris within a grating compressor. The compressor enables the precise tuning of the pulse duration at the specified transition point between LMO system and fiberlink distribution.

To enable an ultra-low residual jitter performance, the lasers are phase-locked to an external microwave oscillator at a comparison frequency of $1.3 \mathrm{GHz}$. This however leaves 24 respectively 6 possible positions where the PLL can catch. The solution to this problem is the introduction of a second PLL into the system running at a comparison frequency which is equal to the repetition rate of the LMO. This leaves only one zero-crossings where the PLL can catch per revolution of the laser pulse inside the cavity. The lower frequency PLL catches first and thus selects the phase of the laser pulse. Once the lock is established, the second PLL running at $1.3 \mathrm{GHz}$ will take over. The switching is done within the digital controller. Once lock of PLL 1 has been established, the ADC signal from PLL 2 will be switched to the DAC. At FLASH, a reference frequency of $9 \mathrm{MHz}$ is supplied identifying the electron bunch bucket. This frequency is multiplied by 6 and compared to the fundamental of the laser in a digital phase detector. The $1.3 \mathrm{GHz}$ machine reference is compared to the filtered harmonic of the laser pulses in a double balanced mixer and the resulting DC phase error is amplified in a low-noise preamplifier to match the ADC input range. The phase detector unit has been constructed and initial performance tests have been conducted.

Digital Controller The digital PI controller was implemented using an in-house developed controller board for the low-level RF control, called SIMCON 3.1 [7]. The application utilizes the Virtex 2 Pro FPGA, located on the controller. The digital controller offers the same functionality as its analog pendant, namely a parallel PI-controller with an optional second order infinite impulse response low-pass filter. The error signal from the double balanced mixer is sampled with a frequency of $50 \mathrm{MHz}$. Then the data is decimated to provide one valid sample every $1 \mu \mathrm{s}$. Optionally averaging of 50 samples of the error signal can be used to reduce the ADC noise. The controller transfer function is applied to the resulting digital signal which is then converted with a DAC and fed back to the piezo via a piezo driver. For diagnostic purposes, a versatile data acquisition system was used. It saves 16 signals over 64 ms (64000 samples of each signal) to the external SRAM memory of the SIMCON controller. Two forms of communication were implemented: a VME interface and an 
RS232 serial link.

Figure 3 shows the phase noise measured when the system is locked for either analog or digital controller. The integration of these signal yields the respective residual jitter. It amounts to $74 \mathrm{fs}$ for the analog controller and $107 \mathrm{fs}$ for the digital controller, both in a bandwidth from $1 \mathrm{~Hz}$ to 112 $\mathrm{kHz}$. The difference in performance of the two controllers is almost entirely due to the PLL overshoot of the digital controller, which can easily be overcome by adjusting the regulation parameters. It is hence possible to achieve comparable jitter performances with a digital regulation compared to its analogue pendant.

The sampling rate of the ADC is a lot higher than needed for the regulation $(60 \mathrm{MHz})$, so an average bringing the effective sampling rate down to $1 \mathrm{MHz}$ was employed. This reduces the uncorrelated noise of the ADC by a factor of almost 8 .

It is important, that the activated PLL does not increase the phase noise at higher offset frequencies. There is no significant increase in the high frequency phase noise when locking with either system as can be seen in figure 3 .

A further important issue is the possible change of the spectral properties of the laser pulses due to the phase locking. The insert to Figure 3 shows the optical spectrum of the fiber laser without any feedback and with either controller. No significant change was observed for either feedback option.

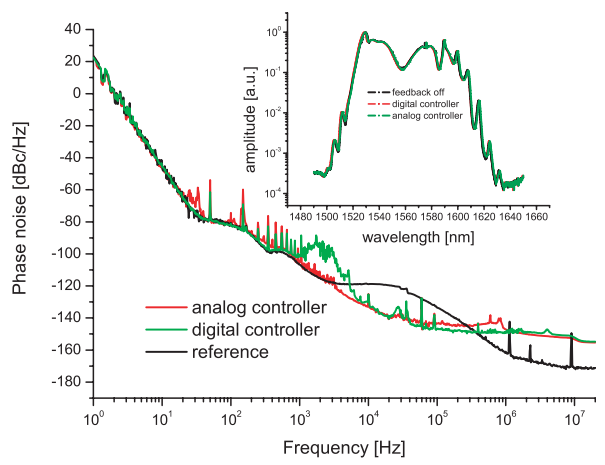

Figure 3: Phase noise for the locked fiber laser with both analog (red) and digital (green) controllers and the reference (black). Insert: Optical spectrum of the fiber laser without feedback (black), with digital controller (red) and analog controller (green)

Diagnostics Five independent parameters will be monitored continuously to ensure proper operation of the lasers. Firstly, a grating and InGaAs camera system is used to monitor the optical spectrum of the lasers. A regular InGaAs photodiode is used to extract the suitable harmonic for the regulation and to monitor the average output power of the laser. The third diagnostic system is a two-photon absorption silicon diode ( $\mathrm{Si}$ is sensitive at $775 \mathrm{~nm}$ ). As this is a nonlinear process which is sensitive to pulse energy, it is a measure of the optical pulse peak power. Furthermore, a cross-correlation of the pulses will be taken and the 07 Accelerator Technology Main Systems high-frequency phase noise will be determined from a spectral line of the photodetected pulse train.

There are three basic failure scenarios which are foreseen to happen. Firstly, the laser can loose mode-lock completely. This is detected in any of the three monitor systems and the FPGA controlling the RF feedback will initialize a swap of the laser system by closing and opening the shutters accordingly. A second scenario is the appearance of a cw-line in the optical spectrum. This means that some energy within the laser cavity is transferred from the single optical pulse circulating inside the cavity into cw background radiation. This can be seen directly in the optical spectrum or even faster in the pulse peak power. Again the FPGA will invoke a switch to the backup system. Thirdly, a change of mode-lock state could occur which is not measurable in spectrum and power but has a negative effect on the phase noise performance, which is an extremely sensitive measurement of the quality of the mode-locking. This is again detected by an ADC channel of the FPGA board and the software acts accordingly, if the jitter value is out of spec.

\section{CONCLUSION AND OUTLOOK}

The proposed layout of the LMO system for FLASH has been given. The $54 \mathrm{MHz}$ fiber lasers have been constructed and initial tests on the phase detector unit and digital PLL have been performed. The setup of the diagnostics will be finalized within the next few months and long-term evaluation will be performed.

\section{REFERENCES}

[1] J. W. Kim et. al., "Large scale timing distribution and RFsynchronization for FEL facilities," FEL Conference 2004, Trieste, Italy, 2004.

[2] A. Winter, F. Loehl, F. Ludwig, H. Schlarb, B. Schmidt, P. Schmueser, "Layout of the optical synchronization system for FLASH," Proceedings of the EPAC 2006, Edinburgh, UK.

[3] A. Winter et. al., "High-precision Laser Master Oscillators for Optical Timing Distribution Systems in Future Light Sources," EPAC Conference 2006, Edinburgh, UK, 2006.

[4] J. Chen, J. W. Sickler, E. P. Ippen, F. X. Kaertner, "High repetition rate, low jitter, low intensity noise, fundamentally mode-locked 167 fs soliton Er-fiber laser,' Optics Letters 32 p. $1566-1568$ (2007).

[5] F. Loehl et. al., "Sub-10 Femtosecond Stabilization of a Fiber-Link using a Balanced Optical Cross Correlator," this conference.

[6] F. Loehl, K. Hacker, H. Schlarb, "A Sub-50 Femtosecond bunch arrival time monitor system for FLASH," Proceedings of the DIPAC 07 Conference, Venice, Italy.

[7] W. Giergusiewicz et. al., "Low latency control board for LLRF system SIMCON 3.1," Proc. of SPIE Vol. 5948, (2005). 